\title{
Video Article \\ Isolation of Papillary and Reticular Fibroblasts from Human Skin by Fluorescence-activated Cell Sorting
}

\author{
Ana Korosec ${ }^{* 1}$, Sophie Frech ${ }^{* 1}$, Beate M. Lichtenberger ${ }^{1}$ \\ ${ }^{1}$ Skin, Endothelium Research Division, Department of Dermatology, Medical University of Vienna \\ *These authors contributed equally
}

Correspondence to: Beate M. Lichtenberger at beate.lichtenberger@meduniwien.ac.at

URL: https://www.jove.com/video/59372

DOI: doi:10.3791/59372

Keywords: Genetics, Issue 147, FACS, cell surface markers, fibroblast heterogeneity, fibroblast lineages, human skin, papillary and reticular fibroblasts

Date Published: 5/7/2019

Citation: Korosec, A., Frech, S., Lichtenberger, B.M. Isolation of Papillary and Reticular Fibroblasts from Human Skin by Fluorescence-activated Cell Sorting. J. Vis. Exp. (147), e59372, doi:10.3791/59372 (2019).

\section{Abstract}

Fibroblasts are a highly heterogeneous cell population implicated in the pathogenesis of many human diseases. In human skin dermis, fibroblasts have traditionally been attributed to the superficial papillary or lower reticular dermis according to their histological localization. In mouse dermis, papillary and reticular fibroblasts originate from two different lineages with diverging functions regarding physiological and pathological processes and a distinct cell surface marker expression profile by which they can be distinguished.

Importantly, evidence from explant cultures from superficial and lower dermal layers suggest that at least two functionally distinct dermal fibroblasts lineages exist in human skin dermis as well. However, unlike for mouse skin, cell surface markers enabling the discrimination of different fibroblast subsets have not yet been established for human skin. We developed a novel protocol for the isolation of human papillary and reticular fibroblast populations via fluorescence-activated cell sorting (FACS) using the two cell surface markers Fibroblast Activation Protein (FAP) and Thymocyte antigen 1 (Thy1)/CD90. This method enables the isolation of pure fibroblast subsets without in vitro manipulation, which was shown to affect gene expression, thus permitting accurate functional analysis of human dermal fibroblast subsets in regard to tissue homeostasis or disease pathology.

\section{Video Link}

The video component of this article can be found at https://www.jove.com/video/59372/

\section{Introduction}

As the main cellular component of connective tissue, fibroblasts are primarily responsible for the deposition of collagen and elastic fibers that ultimately form the extracellular matrix ${ }^{1}$, and thus, their role in tissue homeostasis, regeneration and disease has been underestimated for a long time. However, fibroblasts have recently come under the spotlight of researchers, not only because they represent a prominent cellular source for induced pluripotent stem cells ${ }^{2}$ but also because of their high plasticity and implication in the pathogenesis of a wide number of diseases such as organ fibrosis ${ }^{3,4,5}$ or cancer ${ }^{6,7}$.

Human skin is composed of a multi-layered epithelium, the epidermis, and its underlying connective tissue, the dermis, which can be histologically subdivided into the upper papillary and the lower reticular dermis and is mainly composed of fibroblasts and extracellular matrix ${ }^{8}$ and the hypodermis. According to their location within the tissue, dermal fibroblasts have roughly been classified into papillary and reticular fibroblasts

Importantly, recent data indicate that these dermal fibroblast subpopulations are not only histologically distinguishable, but also that their function is considerably diverse. In mouse skin, papillary and reticular fibroblasts arise from two distinct lineages during embryogenesis ${ }^{9}$. Several lines of evidence suggest that the two lineages exert different roles not only in tissue homeostasis, hair follicle morphogenesis, wound repair and fibrosis $^{7,9,10}$, but they also respond to different signals from neoplastic epidermal stem cells ${ }^{11}$, suggesting diverging roles in cancer pathogenesis. Conveniently, both lineages express a distinct set of mutually exclusive cellular markers in adult mouse skin, thus enabling the isolation of pure dermal fibroblast populations and subsequent extensive analysis of their specific functions in vitro ${ }^{9,11}$

Correspondingly, at least two distinct fibroblast subsets with distinct morphology and functions, including diverging proliferation rates, tissue remodeling capacities ${ }^{12,13}$, as well as the ability to support the growth of epidermal stem cells in vitro, have been described for human skin dermis $^{14,15}$. However, most of the published studies on human dermal fibroblasts have been conducted using mixed fibroblast populations isolated from explant cultures from dermatomed skin, since specific cell surface marker sets enabling the isolation of pure human papillary or reticular fibroblast subpopulations in analogy to mouse dermis were yet to be established. 
We have recently demonstrated, that human skin papillary and reticular fibroblasts are characterized by specific cell surface markers that enable the isolation of the respective subpopulations via fluorescence-activated cell sorting (FACS $)^{16}$ : FAP ${ }^{+} \mathrm{CD}^{-} 0^{-}$fibroblasts represent papillary fibroblasts primarily located in the upper dermis, presenting higher proliferation rates, a distinct gene signature but no adipogenic potential. $\mathrm{FAP}^{+} \mathrm{CD} 90^{+}$and $\mathrm{FAP}^{-} \mathrm{CD} 90^{+}$fibroblasts belong to the reticular lineage of the lower dermal compartments, which are less proliferative but readily undergo adipogenesis-a hallmark for reticular fibroblasts. This method enables to extensively study these distinct fibroblast subpopulations not only in regard to their specific functions under physiological conditions but also in the context of the pathogenesis of cutaneous diseases including skin cancer.

However, since fibroblasts alter their cell surface marker expression in two-dimensional in vitro culture ${ }^{16,17,19}$, the application of our protocol is limited to the isolation of primary fibroblasts from human dermis and does not permit the identification of papillary or reticular fibroblasts in mixed cell culture populations. Importantly, although the expression of cell surface markers changes in vitro, we have demonstrated that the fibroblast subsets isolated according to the protocol described below retain their specific functionality when cultivated ${ }^{16}$, thus enabling in vitro studies of subset-specific properties under physiological or pathological conditions.

In conclusion, we developed a protocol for the isolation of distinct fibroblast subsets via FACS that for the first time permits the isolation of pure fibroblast populations from human skin dermis in a naïve state.

\section{Protocol}

Human skin was obtained from Caucasian men and women aged 26 to 61 years (sun-protected skin; abdominal corrections, breast reductions). Written informed patient consent was obtained before tissue collection in accordance with the Declaration of Helsinki, and with approval from the Vienna Medical University Institutional Review Board.

NOTE: We provide a protocol for the isolation of functionally distinct fibroblast subpopulations either from full thickness dermis (please refer to section 1) or after sectioning human skin dermis into its different layers (skip section 1 and directly refer to section 2).

\section{Preparation of Full Thickness Dermis}

1. Place a $10 \mathrm{~cm} \times 10 \mathrm{~cm}$ human skin piece (e.g., from abdominal corrections or breast reductions) with the epidermis facing downwards on thick filter paper.

2. Hold the epidermis tightly on its edges with forceps and scrape off the subcutaneous fat layer with a scalpel. Then slice the tissue into $5 \mathrm{~mm}$ wide strips before putting them into a Petri dish.

NOTE: This facilitates penetration of Dispase II (referred to as dissociating enzyme henceforth, see section 3 ) and is used if the epidermis needs to be separated from the entire dermis. For this skip section 2 and directly refer to section 3 . To avoid contamination, keep the tissue sterile after surgical removal or clean it thoroughly with ethanol or iodine solution. If at all, ethanol treatment of the skin surface causes only minor cell death. Work under sterile conditions in a tissue culture flow hood.

\section{Sectioning of Human Skin Dermis into Papillary and Reticular Layers with a Dermatome}

1. Place a $10 \mathrm{~cm} \times 10 \mathrm{~cm}$ skin piece (e.g., from abdominal corrections or breast reductions) on thick filter paper, with the epidermis facing upwards. Hold the skin tightly on its edges with forceps.

2. Slice the skin with an electric dermatome, adjusted to a cutting thickness/depth of $300 \mu \mathrm{m}$, by sliding the head of the dermatome away from oneself, with the blade being at a $90^{\circ}$ angle in relation to the surface of the skin.

3. Take the first layer consisting of epidermis and papillary dermis (300 $\mu \mathrm{m}$ thick, "Dermal layer 1", Figure 1 and Figure 2) and put it in a Petri dish. Proceed immediately to section 3 or add $1 \times$ PBS to keep the tissue from drying out.

NOTE: To avoid contamination, keep tissue sterile after surgical removal or clean it thoroughly with ethanol or iodine solution. Work under sterile conditions in a tissue culture flow hood.

CAUTION: Handle dermatome carefully since the blades are very sharp.

4. Repeat step 2.2 adjusting the dermatome to a cutting thickness of $700 \mu \mathrm{m}$ and slice the remaining dermis. Place the upper slice which is defined as the upper reticular dermis ("Dermal layer 2", Figure 2) into a separate Petri dish. Proceed immediately to section 4 or add 1x PBS to keep the tissue from drying out.

5. Scrape away the subcutaneous fat layer with a scalpel from the residual lower reticular dermal layer $(>1,000 \mu \mathrm{m})$ and discard it. Collect the lower reticular dermis ("Dermal layer 3", Figure 2) in another Petri dish. Proceed immediately to section 4 or add 1x PBS to keep the tissue from drying out.

NOTE: Alternatively, instead of scraping the fat off with a scalpel, remove the fat layer with scissors. It may help to put the reticular dermis on ice prior to scraping the fat away.

\section{Separation of Epidermis and Dermis}

1. Prepare a $3 \mathrm{U} / \mathrm{mL}$ dissociating enzyme (i.e., Dispase II) solution in sterile $1 x$ PBS. Place the $5 \mathrm{~mm}$ skin stripes (from section 1), or the epidermis/papillary dermis (from step 2.2), with the epidermis facing upward in a $10 \mathrm{~cm}$ Petri dish with $10 \mathrm{~mL}$ of $3 \mathrm{U} / \mathrm{mL}$ dissociating enzyme solution. Incubate the Petri dish at $37^{\circ} \mathrm{C}$ for $1 \mathrm{~h}$.

1. The protocol may be paused here. Incubate epidermis/papillary dermis in protease in the refrigerator at $4{ }^{\circ} \mathrm{C}$ overnight instead. As needed store the other layers (Dermal layer 1,2 and 3) overnight immersed in $1 \times$ PBS in $50 \mathrm{~mL}$ tubes at $4{ }^{\circ} \mathrm{C}$.

CAUTION: Work carefully with protease, it may irritate skin and eyes upon contact. 
2. After incubation, transfer the epidermis/papillary dermis to the dry lid of the Petri dish and separate the epidermis from the upper dermis (Dermal layer 1) with two forceps each holding the edge of either the epidermis or dermis.

\section{Enzymatic Digestion of Dermal Tissue}

1. Mince each dermal layer (Dermal layer 1,2 and 3) separately with scissors/scalpel as thoroughly as possible; the smaller the pieces, the better the tissue digestion.

NOTE: The toughness of the dermis can vary depending on the body part it originates from (e.g., abdominal or upper arm skin).

2. Prepare the digestion mix by combining $1.25 \mathrm{mg} / \mathrm{mL}$ collagenase $I, 0.5 \mathrm{mg} / \mathrm{mL}$ collagenase $I I, 0.5 \mathrm{mg} / \mathrm{mL}$ collagenase IV and $0.1 \mathrm{mg} / \mathrm{mL}$ hyaluronidase in Dulbecco's modified Eagle's medium (DMEM) with L-glutamine (see Table of Materials), $10 \%$ fetal calf serum (FCS) and $1 \%$ penicillin/streptomycin (P/S).

CAUTION: Work carefully with collagenases as these may irritate skin and eyes upon contact.

3. Put each of the minced tissues with $10 \mathrm{~mL}$ of the prepared digestion mix into a $50 \mathrm{~mL}$ tube and incubate in a $37^{\circ} \mathrm{C}$ warm water bath for $1 \mathrm{~h}$ under permanent agitation. During digestion invert the tubes several times.

NOTE: One should consider adjusting the digestion volume according to the size of the minced skin piece. Do not overload $10 \mathrm{~mL}$ digestion mix with too much tissue otherwise the cell yield will be minimal. Less than one third of tissue within the total volume is recommended (1:2 w/ v).

\section{Preparation of Single Cell Suspension and Erythrocyte Lysis}

1. Stop enzymatic tissue digestion by adding $10 \mathrm{~mL}$ fibroblast medium (DMEM with L-glutamine, $10 \%$ FCS and $1 \% \mathrm{P} / \mathrm{S}$ ) to the digested tissue. Work on ice from here on.

2. Pour the contents of each tube through a regular sterile stainless tea strainer and collect cell suspension in a clean Petri dish. Wash the strainer with medium and mash undigested tissue pieces with the edge of a syringe-plunger.

3. Afterwards, pipette collected cell suspension through a $70 \mu \mathrm{m}$ cell strainer into a $50 \mathrm{~mL}$ tube. Rinse the cell strainer with medium and collect flow through into the same tube.

4. Centrifuge tubes at $500 \times g$ at $4{ }^{\circ} \mathrm{C}$ for $10 \mathrm{~min}$. Remove and discard supernatant and wash cell pellet with $5 \mathrm{~mL}$ fibroblast medium. Repeat the centrifugation step.

5. Remove and discard the supernatant and resuspend the pellet in $1 \mathrm{~mL}$ self-made $A C K$ erythrocyte lysis buffer $\left(0.15 \mathrm{M} \mathrm{NH}{ }_{4} \mathrm{Cl}, 10 \mathrm{mM}\right.$ $\left.\mathrm{KHCO}_{3}, 0.1 \mathrm{mM} \mathrm{Na}_{2} \mathrm{EDTA} ; \mathrm{pH} 7.2-7.4\right)$. Leave cells in lysis buffer for approximately $1 \mathrm{~min}$ at ambient temperature $\left(20-22^{\circ} \mathrm{C}\right)$.

6. Stop lysis by adding $9 \mathrm{~mL}$ of $1 \times$ PBS with $10 \% \mathrm{FCS}$ and centrifuge the tubes again at $500 \times \mathrm{g}$ at $4{ }^{\circ} \mathrm{C}$ for 5 min. Discard the supernatant. CAUTION: The incubation time in erythrocyte lysis may be adjusted if the lysis seems incomplete (red pellet). However, do not leave the cells in erythrocyte lysis buffer for too long to avoid lysis of fibroblasts and immune cells.

\section{Blocking and Staining Fibroblasts for FACS}

1. Prepare $5 \mu \mathrm{L}$ of human Fc-receptor blocking solution in $100 \mu \mathrm{L}$ of $1 \times$ PBS with $10 \%$ FCS and resuspend cells in $100 \mu \mathrm{L}$ of human Fc blocker. Incubate on ice for $10 \mathrm{~min}$.

2. Design a FACS staining panel to stain human dermal fibroblasts. Mix anti-human FAP APC (1:20), anti-human CD90 AF700 (1:30), antihuman E-cadherin PE (1:20), anti-human CD31 FITC (1:30), anti-human CD45 Pacific blue (1:20), anti-human ITGA6 PE (1:20), anti-human CD235ab Pacific blue $(1: 1,000)$ and anti-human CD106 Pacific blue $(1: 100)$ in 1x PBS with 10\% FCS.

3. After Fc receptor blocking, spin cells down at $500 \times \mathrm{g}$ at $4{ }^{\circ} \mathrm{C}$ for $3 \mathrm{~min}$. Remove and discard the supernatant and resuspend cells in $100 \mu \mathrm{L}$ of antibody mix. Incubate cells in the dark at $4{ }^{\circ} \mathrm{C}$ for $20 \mathrm{~min}$.

1. Prior to staining, remove $20 \mu \mathrm{L}$ of a sample with a high cell number for unstained and single stain FACS controls.

4. Wash the stained cells and FACS controls twice with $500 \mu \mathrm{L} 1 \times \mathrm{PBS}$ with $10 \% \mathrm{FCS}$ and centrifuge at $500 \times \mathrm{g}$ at $4{ }^{\circ} \mathrm{C}$ for $3 \mathrm{~min}$. In the meantime, add 4',6-diamidino-2-phenylindole (DAPI) to $1 \times$ PBS with $10 \%$ FCS to make a final concentration of $1 \mu \mathrm{g} / \mathrm{mL}$.

5. Remove and discard supernatant, resuspend cells in $200 \mu \mathrm{L}$ DAPI solution and filter each cell suspension through a $70 \mu \mathrm{m}$ cell strainer into a $5 \mathrm{~mL}$ FACS tube.

NOTE: If FACS is going to be performed with delay, add DAPI and filter shortly before recording.

\section{Gating Strategy for Human dermal Fibroblast Subset Isolation and FACS}

1. Record flow cytometry controls, set correct voltage settings and perform the appropriate compensation. Gate for single cells and live (DAPI'), Pacific blue, PE and FITC negative cells (CD45, CD31', CD235ab, CD106", ITGA6 and E-cadherin ) to get three fibroblast populations: $\mathrm{FAP}^{+} \mathrm{CD} 90^{-}, \mathrm{FAP}^{+} \mathrm{CD} 90^{+}$and $\mathrm{FAP}^{-} \mathrm{CD} 90^{+}$cells. See Figure 3.

2. Sort three fibroblast subpopulations into separate $1.5 \mathrm{~mL}$ screw cap microcentrifuge tubes filled either with $350 \mu \mathrm{L}$ lysis buffer for RNA isolation or filled with $350 \mu \mathrm{L}$ fibroblast growth medium (see the Table of Materials) for cell culture. Invert tubes immediately after the sort and either put lysis buffer tubes into liquid nitrogen for snap freezing or put medium tubes on ice.

CAUTION: Prepare lysis buffer with $0.1 \%$ b-Mercaptoethanol in fume hood and avoid inhalation.

\section{Culturing of Fibroblasts and Adipogenesis Assay}

1. Following FACS, spin cells down at $500 \times g$ for $3 \mathrm{~min}$ at $4{ }^{\circ} \mathrm{C}$ and plate equal cell numbers $(5,000-10,000$ cells/well) in fibroblast growth medium (see the Table of Materials) in 48-well cell culture dishes. Leave cells to grow until they reach $70 \%$ confluency.

2. Add $5 \mu \mathrm{g} / \mathrm{mL}$ insulin, $5 \mu \mathrm{M}$ troglitazone, $0.5 \mathrm{mM}$ 3-isobutyl-1-methylxanthine (IBMX) and $1 \mu \mathrm{M}$ dexametasone to fibroblast growth medium to prepare adipocyte differentiation medium. 
3. Replace medium of the cultured cells with adipocyte differentiation medium and let cells differentiate for 14 days. Exchange medium after day 2 with reduced adipogenesis medium containing $5 \mu \mathrm{g} / \mathrm{mL}$ insulin and $5 \mu \mathrm{M}$ troglitazone. Replenish medium every $3^{\text {rd }}$ or $4^{\text {th }}$ day.

4. Fix cells with $4 \%$ PFA for 20 min at ambient temperature $\left(20-22{ }^{\circ} \mathrm{C}\right)$ at differentiation day 14 . Wash wells with $60 \%$ isopropanol and let it evaporate completely. Stain cells with $5 \mathrm{mM}$ Oil Red O (filter prior to use) for $20 \mathrm{~min}$. Wash stained cells four times with distilled water. Proceed to perform microscopy.

CAUTION: PFA is toxic and harmful. Handle carefully.

NOTE: The protocol can be paused here. Fixed cells can be stored in $1 \times \mathrm{PBS}$ at $4{ }^{\circ} \mathrm{C}$ until Oil Red $\mathrm{O}$ staining is performed. Cover dish with paraffin film prior to storage to avoid evaporation.

5. Image cells immersed in water with an inverted microscope at 10x magnification with transmitted light.

\section{Representative Results}

An overview of the main steps for processing skin tissue to obtain a single cell suspension is shown in Figure 1, displaying the dermatoming of the skin (Figure 1A), different dermal layers (Figure 1B), removal of the subcutaneous fat layer (Figure 1C) and the separation of the epidermis and papillary dermis (Figure 1D), as well as the different steps of the manual and enzymatic tissue dissociation (Figure 1E,F). A scheme of the three dermal layers is provided in Figure 2.

Figure 3 displays the gating strategy of a flow cytometry staining panel for the analysis of different fibroblast subsets from human skin. Additional cell surface markers that are not expressed on fibroblasts permit the exclusion of various other cells present in the skin such as immune cells, epidermal cells, mesenchymal stem cells (MSCs), red blood cells or endothelial cells to attain maximal purity in the isolated populations. It is not critical to use the identical FACS panel used in Figure $\mathbf{2}$ for the identification of these fibroblast subpopulations, however this is our recommendation. One may use antibodies labelled with different fluorescent dyes or alter the combination of exclusion markers.

Of note, this protocol enables the identification of three fibroblast populations in human skin which present with different intradermal localization, gene expression profiles and also functions (Figure 4). $\mathrm{FAP}^{+} \mathrm{CD} 90^{-}$are enriched in the papillary dermis whereas $\mathrm{FAP}^{+} \mathrm{CD} 90^{+}$and $\mathrm{FAP}^{-} \mathrm{CD} 90^{+}$are more abundant in the reticular dermis (Figure 4A). All three subpopulations exhibit the typical fibroblast morphology upon sorting in cell culture (Figure 4B). Interestingly, they differ regarding their ability to differentiate into adipocytes which is a hallmark for reticular fibroblasts. Combining these results with gene expression profiling via real-time polymerase chain reaction (RT-PCR) ${ }^{16}$ (Figure $4 \mathrm{C}$ ), showing that FAP $^{+}$CD90 cells express high levels of markers commonly attributed to papillary fibroblasts such as CD26, NTN and PDPN while CD90 ${ }^{+}$cells express the known reticular markers such as CD36, ACTA2 and PPARy at high levels, we conclude that FAP ${ }^{+} \mathrm{CD} 90^{-}$cells belong to the papillary and $\mathrm{CD} 90^{+}$cells belong to the reticular lineage.
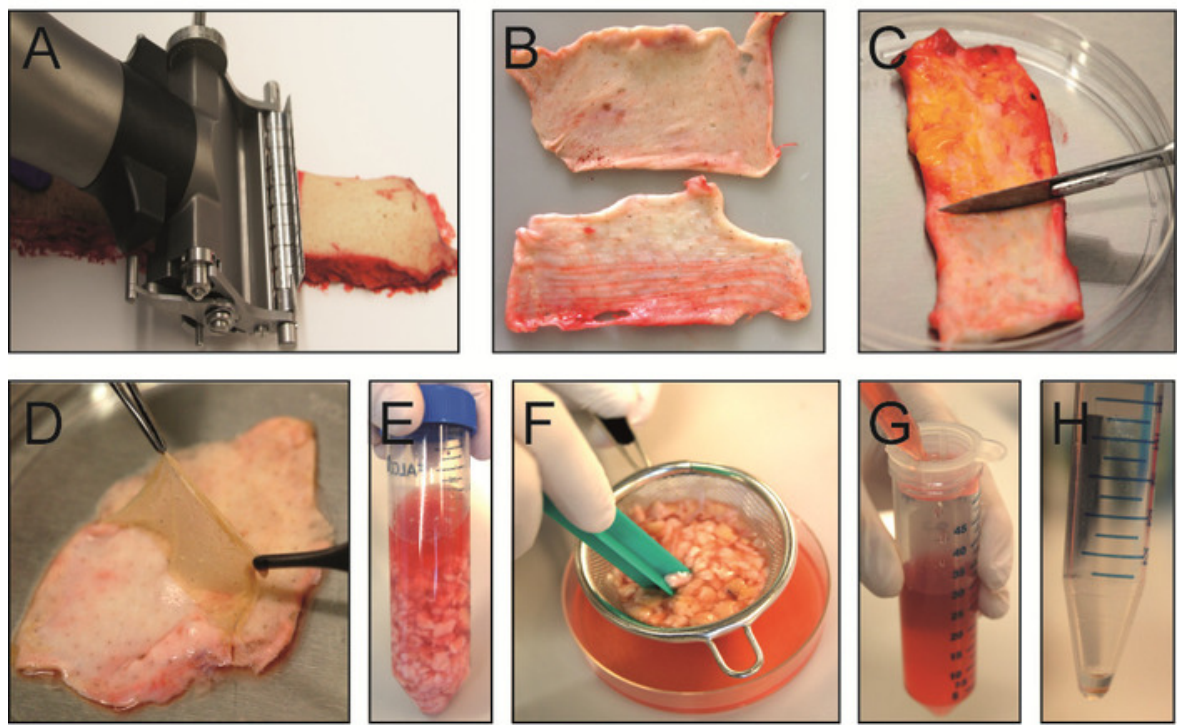

Figure 1: Isolation of dermal single cell suspension from intact human skin. (A) Skin is sliced with an electric dermatome into papillary and reticular dermis. (B) Papillary dermis with adjacent epidermis is $300 \mu \mathrm{m}$ thick (top) while upper reticular dermis is $700 \mu \mathrm{m}$ thick (bottom). (C) Subcutaneous fat layer is scraped-off of lower reticular dermis with a scalpel. (D) After incubation of papillary dermis in dissociation enzyme solution at $37^{\circ} \mathrm{C}$ for $1 \mathrm{~h}$, the epidermis can easily be removed with forceps. (E) Different dermal layers are minced with scissors and transferred into an enzyme digestion mix consisting of collagenase I, II and IV and hyaluronidase. (F) After $1 \mathrm{~h}$ of dissociation at $37^{\circ} \mathrm{C}$, tissue digestion is stopped and the suspension is poured through a tea strainer to remove undigested skin pieces. (G) Cell suspension is filtered once more through a $70 \mu \mathrm{m}$ cell strainer and centrifuged at $500 \times \mathrm{g}$ at $4{ }^{\circ} \mathrm{C}$ for $10 \mathrm{~min}$. $(\mathbf{H})$ After centrifugation, the cell pellet is washed with $1 \times$ PBS with $10 \%$ FCS and is ready for FACS staining. Please click here to view a larger version of this figure. 


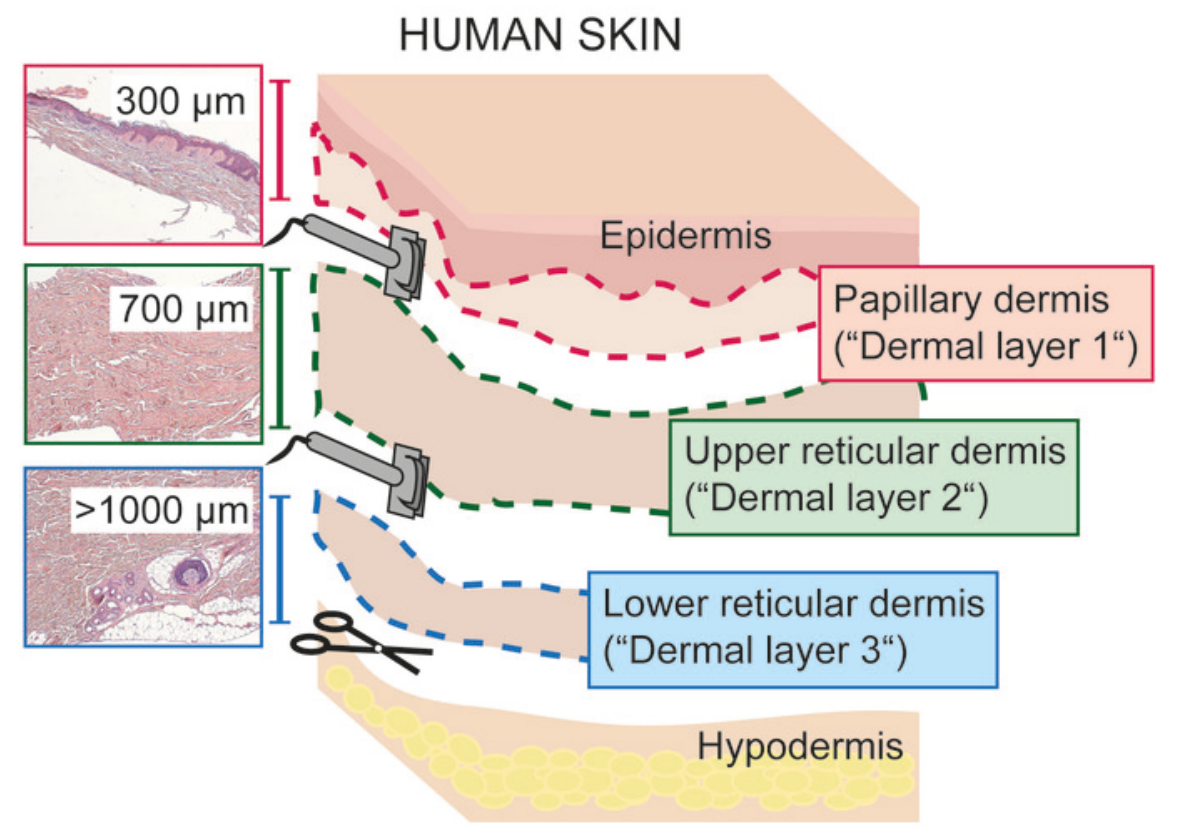

Figure 2: Sectioning of human skin dermis into papillary and reticular layers with a dermatome. Scheme showing the three dermal layers obtained by slicing full thickness skin into papillary dermis (including epidermis; 0-300 $\mu \mathrm{m}$; dermal layer 1), upper reticular (300-1,000 $\mu \mathrm{m}$; dermal layer 2$)$ and lower reticular dermis $(>1,000 \mu \mathrm{m}$; dermal layer 3$)$ with a dermatome. Please click here to view a larger version of this figure.
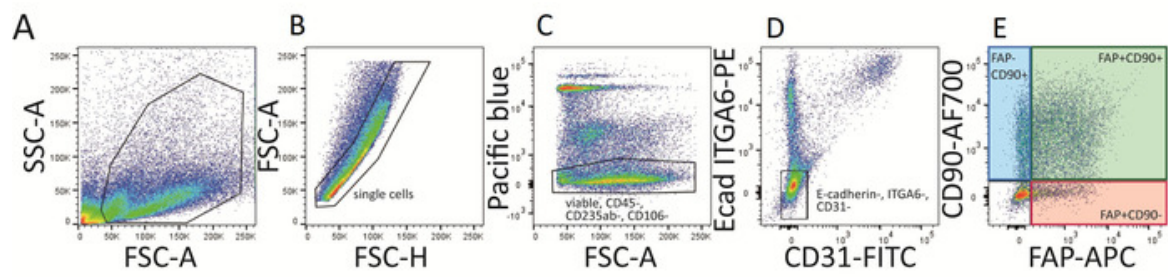

Figure 3: FACS gating strategy for human dermal fibroblast subpopulations. (A-E) First, cells are gated on single (B) and viable (DAPI') cells $(\mathbf{C})$. Immune cells $\left(\mathrm{CD} 45^{+}\right)$, mesenchymal stem cells $\left(\mathrm{CD} 106^{+}\right)$, red blood cells $\left(\mathrm{CD} 235 \mathrm{ab}^{+}\right)$are excluded $(\mathbf{C})$ and Pacific blue negative cells are gated further on E-cadherin (Ecad) and ITGA6 double negative cells (PE channel, D). E-cadherin and ITGA6 are markers expressed on epidermal cells. Next, CD31-FITC positive cells (endothelial and lymphatic cells) are excluded (D) resulting in three fibroblast populations expressing either one or both of the two cell surface fibroblast markers FAP and CD90: FAP ${ }^{+} \mathrm{CD}^{-}, \mathrm{FAP}^{+} \mathrm{CD}^{+} 0^{+}$and FAP $\mathrm{CD}^{-} \mathrm{C}^{+}(\mathrm{E})$. Please click here to view a larger version of this figure. 
A

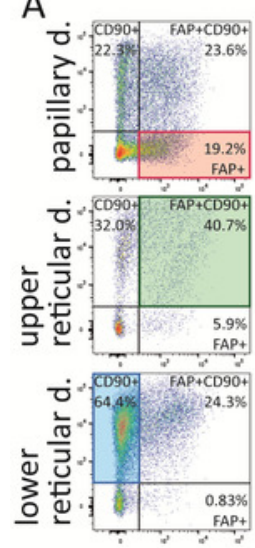

B
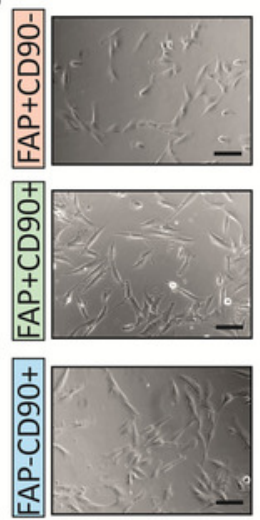
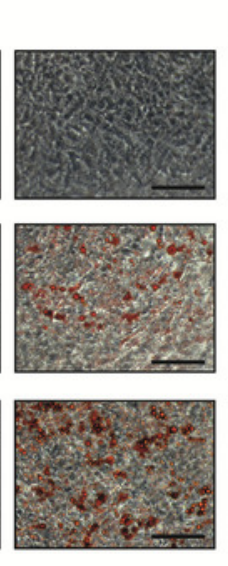

$\mathrm{C}$

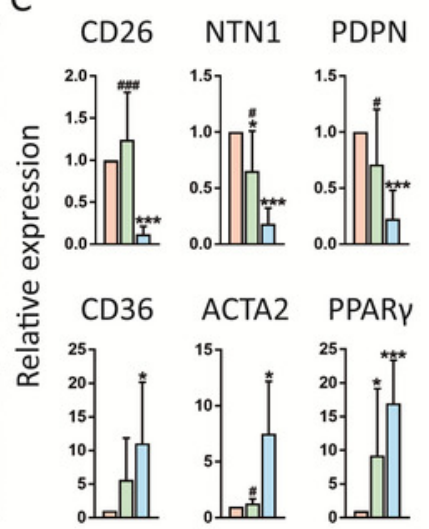

Figure 4: $\mathrm{FAP}^{+} \mathrm{CD} 90^{-}, \mathrm{FAP}^{+} \mathrm{CD}^{+}$and $\mathrm{FAP}^{-} \mathrm{CD}^{\circ} 0^{+}$fibroblasts differ in dermal localization, gene expression and functionality. (A) Representative FACS plots of gated fibroblasts isolated from papillary, upper reticular and lower reticular dermis. Gating strategy is explained in Figure 3. $\mathrm{FAP}^{+} \mathrm{CD} 90^{-}$fibroblasts are enriched in the papillary dermis $(19.2 \%)$ compared to lower reticular dermis $(0.83 \%)$. $\mathrm{FAP}^{+} \mathrm{CD}^{+} 0^{+}$ cells can be found throughout the dermis but their highest abundance is in the upper reticular dermis $(40.7 \%)$. FAP ${ }^{-} \mathrm{CD}^{+} 0^{+}$are enriched in the lower reticular dermis (64.4\%). (B) Of note, all three sorted subpopulations display typical fibroblast morphology upon culture for 7 days (left). Interestingly, they behave differently in an adipogenesis assay. After 14 days in culture, $\mathrm{FAP}^{+} \mathrm{CD} 90^{-}$do not differentiate into adipocytes while $\mathrm{FAP}^{+} \mathrm{CD} 90^{+}$and $\mathrm{FAP}^{-} \mathrm{CD} 90^{+}$readily undergo adipogenesis (right; Oil Red O stains lipid-bearing cells red). Scale bars $=1,000 \mu \mathrm{m}$. $(\mathrm{C})$ Directly sorted $\mathrm{FAP}^{+} \mathrm{CD} 90^{-}$fibroblasts express the papillary fibroblast markers CD26, NTN1 and PDPN, while FAP ${ }^{-} \mathrm{CD}^{+} 0^{+}$cells express CD36, ACTA2 and PPARy, known to be expressed by the reticular lineage. ${ }^{*} p \leq 0.05 ;{ }^{* * *} p \leq 0.0005$ compared to FAP ${ }^{+} / C^{*} 90^{-}$cells; $^{\#} p \leq 0.05 ;{ }^{\# \#} p \leq 0.0005$ compared to $\mathrm{FAP}^{-} / \mathrm{CD}^{+} 0^{+}$cells. Please click here to view a larger version of this figure.

\section{Discussion}

In this article, we describe a method for the isolation of papillary and reticular fibroblasts from human skin. CD90 has been widely used for the identification or isolation of dermal fibroblasts ${ }^{18,20,21}$. However, we have demonstrated that besides CD90+ fibroblasts, human dermis also harbors a $\mathrm{CD}^{-}$fibroblast population expressing FAP $^{16}$, which has been established as a marker for activated fibroblasts and cancer-associated fibroblasts (CAFs) ${ }^{22,23,24,25}$. Importantly, we were able to identify three fibroblast subpopulations FAP $\mathrm{CD}^{+} 0^{-}, \mathrm{FAP}^{+} \mathrm{CD}^{+} 0^{+}$and $\mathrm{FAP}^{-} \mathrm{CD}^{+} 0^{+}$in skin biopsies from all healthy human donors. We therefore conclude that FAP is not only a marker for activated fibroblasts or CAFs but also normal tissue fibroblasts.

Of note, the $\mathrm{FAP}^{-} \mathrm{CD} 90^{-}$cell population remaining after application of the above-described exclusion- and gating strategy does not contain fibroblasts, since these cells do not proliferate in fibroblast cultivation medium in vitro, but most likely a mixed cell population including lymphatic cells and pericytes amongst others ${ }^{16}$.

The cell yield obtained by use of the above-described protocol can vary depending on the body-part that the skin piece used for the isolation originates from. Dermis from different body parts differs regarding its structure, thickness as well as collagen composition. For example, skin from the face or the upper arm is much thinner than skin from the belly or the thigh, which also frequently display a thicker subcutaneous fat layer. Additionally, age and sex of the skin donors may further not only impact the tissue dissociation efficiency, but may also affect the distribution of the three fibroblast subpopulations (Figure 3) when isolated from full thickness skin. This results from the fact that the papillary dermis shrinks and that total fibroblast numbers decrease with age ${ }^{11,26,27,28}$. Furthermore, the cell pellet from papillary dermis will probably be larger than from reticular dermis, since the upper dermis is more densely populated by fibroblasts than the reticular dermis. Besides, the lower dermis is also tougher and more densely packed with collagen, making it harder to dissociate the tissue and to release the fibroblasts. Of note, the cell pellet may appear very red, which is why red blood cell lysis is recommended.

In addition to the identification of three subpopulations in intact human skin, we also show that in dermatomed skin, each fibroblast subset is enriched either in papillary or reticular dermis ${ }^{16}$. Precise slicing of the skin with the dermatome is critical to obtain a proper enrichment of each subpopulation from different dermal layers. Since the papillary dermis is very thin, the dermatomed slice representing it should not exceed a thickness of $300 \mu \mathrm{m}$. Upper reticular and lower reticular fibroblasts both represent the reticular lineage and display similar functions and gene signatures, therefore, one could also consider not separating them.

Importantly, all three fibroblasts populations are found throughout the dermis and are not exclusively present in one layer, which is why explant cultures from papillary or reticular dermis result in mixed fibroblast cultures. However, FAP ${ }^{+}$CD90 papillary fibroblast are most abundant in the papillary dermis and follow a gradient from superficial to lower dermal layers while $\mathrm{FAP}^{+} \mathrm{CD} 90^{+}$and $\mathrm{FAP}^{-} \mathrm{CD} 90^{+}$fibroblasts follow an inverse gradient from the lower to the superficial layers ${ }^{16}$. Furthermore, the majority of $\mathrm{CD} 0^{+}$fibroblasts of the papillary dermis are almost exclusively found surrounding blood vessels and express the perivascular fibroblast marker CD146 ${ }^{29}$, and thus probably exhibit different functions than the remaining CD146 reticular fibroblasts ${ }^{16}$. CD146 could be used as an additional marker in the gating strategy to exclude this population.

Following the dissociation of dermal layers, the isolated cells are stained with a specially designed antibody cocktail containing various antibodies for the exclusion of immune cells, endothelial and lymphatic cells, epidermal cells, erythrocytes and MSCs to obtain pure fibroblast populations. Of note, choosing a marker for the identification and exclusion of MSCs may be tricky because of the high number of published MSC markers ${ }^{30,31}$. Since MSCs express CD90 like fibroblasts, additional MSC markers such as CD105 or CD271 could prove useful for their 
identification. However, MSCs only represent a very low percentage of all dermal cells and since CD90 fibroblasts display typical morphological features of fibroblasts upon sorting, one could argue that the exclusion of MSCs by use of distinct cell surface markers might be unnecessary.

Importantly, we analyzed FAP and CD90 gene expression after keeping the cells in culture for 7-14 days after sorting (data not shown) and found that expression of both markers is upregulated in the respective sorted single positive $\left(\mathrm{FAP}^{+} \mathrm{CD}^{-} 0^{-}\right.$or FAP $\left.\mathrm{FD}^{-} \mathrm{C}^{+}\right) \mathrm{Cells}^{16}$. We therefore emphasize that the above-described marker sets and protocol permit the isolation of primary fibroblast subsets directly from the tissue but not from previously cultured mixed fibroblast populations.

Nevertheless, we demonstrate that the functionality of all three subpopulations is retained in cell culture regardless of the alteration of cell surface marker expression, since fibroblasts sorted as $\mathrm{FAP}^{+} \mathrm{CD} 90^{-}$papillary fibroblasts do not acquire the ability to undergo adipogenesis after a longer period of culture, while fibroblasts sorted as $\mathrm{FAP}^{+} \mathrm{CD}{ }^{+}$or $\mathrm{FAP}^{-} \mathrm{CD} 90^{+}$reticular fibroblasts maintain their ability to differentiate into adipocytes ${ }^{16}$. Importantly, we also found that papillary and reticular-specific genes are still expressed to a higher extent in FAP $^{+} \mathrm{CD}^{-} 0^{-}$and CD90 ${ }^{+}$respectively.

In conclusion, we have established a protocol for the isolation of functionally distinct fibroblast subsets via FACS that for the first time permits the isolation and analysis of pure and naïve fibroblast subpopulations from human skin dermis. This method establishes a major advancement to the commonly used fibroblast explant culture isolation protocol from upper and lower dermis as (i) an opposing gradient of papillary and reticular fibroblasts exists from the skin surface to the hypodermis and (ii) fibroblasts change their gene signature in vitro.

\section{Disclosures}

The authors have nothing to disclose.

\section{Acknowledgments}

We gratefully acknowledge assistance in FACS-sorting by Bärbel Reininger and Wolfgang Bauer. This work was supported by grants to B. M. L. from the Austrian Science Fund (FWF: V 525-B28), and the Federation of European Biochemical Societies (FEBS, Follow-Up Research Fund). S. F. is recipient of a DOC Fellowship of the Austrian Academy of Sciences (OeAW). We gratefully acknowledge excellent support from the core facilities at the Medical University of Vienna. We thank Bernhard Gesslbauer, Christine Radtke and Martin Vierhapper for providing human skin material.

\section{References}

1. Sorrell, J. M., Caplan, A. I. Fibroblast heterogeneity: more than skin deep. Journal of Cell Science. 117 (Pt 5), 667-675, (2004).

2. Lowry, W. E. et al. Generation of human induced pluripotent stem cells from dermal fibroblasts. Proceedings of the National Academy of Sciences of the United States of America. 105 (8), 2883-2888, (2008).

3. Kisseleva, T. The origin of fibrogenic myofibroblasts in fibrotic liver. Hepatology. 65 (3), 1039-1043, (2017).

4. LeBleu, V. S. et al. Origin and function of myofibroblasts in kidney fibrosis. Nature Medicine. 19 (8), 1047-1053, (2013).

5. Travers, J. G., Kamal, F. A., Robbins, J., Yutzey, K. E., Blaxall, B. C. Cardiac Fibrosis: The Fibroblast Awakens. Circulation Research. 118 (6), 1021-1040, (2016).

6. Kalluri, R., Zeisberg, M. Fibroblasts in cancer. Nat Rev Cancer. 6 (5), 392-401, (2006).

7. Rinkevich, Y. et al. Skin fibrosis. Identification and isolation of a dermal lineage with intrinsic fibrogenic potential. Science. 348 (6232), aaa2151, (2015).

8. Abhishek, S., Palamadai Krishnan, S. Epidermal Differentiation Complex: A Review on Its Epigenetic Regulation and Potential Drug Targets Cell Journal. 18 (1), 1-6, (2016).

9. Driskell, R. R. et al. Distinct fibroblast lineages determine dermal architecture in skin development and repair. Nature. 504 (7479), 277-281, (2013).

10. Mastrogiannaki, M. et al. beta-Catenin Stabilization in Skin Fibroblasts Causes Fibrotic Lesions by Preventing Adipocyte Differentiation of the Reticular Dermis. Journal of Investigative Dermatology. 136 (6), 1130-1142, (2016).

11. Lichtenberger, B. M., Mastrogiannaki, M., Watt, F. M. Epidermal beta-catenin activation remodels the dermis via paracrine signalling to distinct fibroblast lineages. Nature Communications. 7 10537, (2016).

12. Harper, R. A., Grove, G. Human skin fibroblasts derived from papillary and reticular dermis: differences in growth potential in vitro. Science. 204 (4392), 526-527, (1979).

13. Hiraoka, C. et al. Two clonal types of human skin fibroblasts with different potentials for proliferation and tissue remodeling ability. Journal of Dermatological Science. 82 (2), 84-94, (2016).

14. Higgins, C. A. et al. Multifaceted role of hair follicle dermal cells in bioengineered skins. Br Journal of Dermatology. 176 (5), $1259-1269$, (2017).

15. Korosec, A., Lichtenberger, B. M. in Skin Tissue Models. https://doi.org/10.1016/B978-0-12-810545-0.00012-7 eds A. P. Marques, R. P. Pirraco, M. Cerqueira,, R. L. Reis) Ch. 12, 279-301 Elsevier (2017).

16. Korosec, A. et al. Lineage identity and location within the dermis determine the function of papillary and reticular fibroblasts in human skin. Journal of Investigative Dermatology. (2018).

17. Higgins, C. A., Chen, J. C., Cerise, J. E., Jahoda, C. A., Christiano, A. M. Microenvironmental reprogramming by three-dimensional culture enables dermal papilla cells to induce de novo human hair-follicle growth. Proceedings of the National Academy of Sciences of the United States of America. 110 (49), 19679-19688, (2013).

18. Philippeos, C. et al. Spatial and Single-Cell Transcriptional Profiling Identifies Functionally Distinct Human Dermal Fibroblast Subpopulations. Journal of Investigative Dermatology. 138 (4), 811-825, (2018). 
19. Walmsley, G. G. et al. Live fibroblast harvest reveals surface marker shift in vitro. Tissue Engineering Part C: Methods. 21 (3), $314-321$, (2015).

20. Jiang, D., Rinkevich, Y. Defining Skin Fibroblastic Cell Types Beyond CD90. Frontiers in Cell and Developmental Biology. 6 133, (2018).

21. Sorrell, J. M., Caplan, A. I. Fibroblasts-a diverse population at the center of it all. International Review of Cell and Molecular Biology. 276 161-214, (2009).

22. Huang, Y. et al. Isolation of Fibroblast-Activation Protein-Specific Cancer-Associated Fibroblasts. BioMed Research International. 2017 4825108, (2017).

23. Jiang, G. M. et al. The application of the fibroblast activation protein alpha-targeted immunotherapy strategy. Oncotarget. 7 (22), 33472-33482, (2016).

24. Pure, E., Blomberg, R. Pro-tumorigenic roles of fibroblast activation protein in cancer: back to the basics. Oncogene. 37 (32), $4343-4357$, (2018).

25. Hamson, E. J., Keane, F. M., Tholen, S., Schilling, O., Gorrell, M. D. Understanding fibroblast activation protein (FAP): substrates, activities, expression and targeting for cancer therapy. PROTEOMICS - Clinical Applications. 8 (5-6), 454-463, (2014).

26. Gilchrest, B. A. A review of skin ageing and its medical therapy. Br Journal of Dermatology. 135 (6), $867-875$ (1996).

27. Gilchrest, B. A., Krutmann, J. Skin Aging. 198 Springer (2006).

28. Makrantonaki, E., Zouboulis, C. C. Molecular mechanisms of skin aging: state of the art. Annals of the New York Academy of Sciences. 1119 40-50, (2007).

29. Crisan, M. et al. A perivascular origin for mesenchymal stem cells in multiple human organs. Cell Stem Cell. 3 (3), 301-313, (2008).

30. Kundrotas, G. Surface markers distinguishing mesenchymal stem cells from fibroblasts. Acta Medica Lituanica. 19 (2), 75-79, (2012).

31. Lupatov, A. Y., Vdovin, A. S., Vakhrushev, I. V., Poltavtseva, R. A., Yarygin, K. N. Comparative analysis of the expression of surface markers on fibroblasts and fibroblast-like cells isolated from different human tissues. Bulletin of Experimental Biology and Medicine. 158 (4), 537-543, (2015). 